\title{
Performance Assessment of a Distributed Intrusion Detection System in a Real Network Scenario
}

\author{
Salvatore D’Antonio*, Valerio Formicola ${ }^{\dagger}$, Claudio Mazzariello ${ }^{\dagger}$, Francesco Oliviero ${ }^{\dagger}$ and Simon Pietro Romano ${ }^{\dagger}$ \\ ${ }^{*}$ Dipartimento delle Tecnologie - Parthenope University of Napoli, Italy \\ Email: salvatore.dantonio@uniparthenope.it \\ ${ }^{\dagger}$ Dipartimento di Informatica e Sistemistica - Federico II University of Napoli, Italy \\ Email: \{valerio.formicola, cmazzari, folivier, spromano\}@unina.it
}

\begin{abstract}
The heterogeneity and complexity of modern networks and services urge the requirement for flexible and scalable security systems, which can be dynamically configured to suit the everchanging nature of security threats and user behavior patterns. In this paper we present a distributed architecture for an Intrusion Detection System, allowing for traffic analysis at different granularity levels, performed by using the best available techniques. Such architecture leverages the principle of separation of concerns, and hence proposes to build up a system comprising entities specialized in performing different tasks, appropriately orchestrated by a broker entity playing the crucial role of the mediator. This paper stresses the point that a distributed system, besides being inherently more scalable than a centralized one, allows for better detection capabilities thanks to the effective exploitation of the inner heterogeneity of the involved detection engines. In order to support our findings, we will describe the design, implementation and deployment of the proposed solution in the framework of the INTERSECTION FP7 European Project.
\end{abstract}

Index Terms-Network security and protection, Intrusion Detection, Distributed Systems

\section{INTRODUCTION}

Intrusion Detection Systems (IDS) nowadays represent a well-known solution to identify unauthorized and malicious use of the network resources. The relevance of the tasks carried out by an IDS necessarily poses several requirements to be met since the design phase. The first peculiarity of an efficient IDS should be the accuracy of the detection process. Accuracy is related to the capability of the system to both correctly classify ongoing attacks and avoid misclassifications of normal traffic as attacks. In order to improve accuracy, sophisticated mechanisms and algorithms have been proposed. Recently, we have been witnessing the development of innovative solutions which, rather than focusing on the definition of novel detection methods, propose the adoption of already assessed techniques appropriately combined in the framework of a multiple classifier approach [1], [2]. Another important requirement of an IDS is its scalability. Since the volume of network traffic to be analyzed might keep on growing as time elapses, an efficient IDS should be capable of supporting correctly this increased load, usually by allocating new resources to the computation process. Ideally the amount of the needed additional resources should be a linear function of the increase in network traffic load. Since more and more novel attacks threaten the security of networks and, at the same time, new methodologies are proposed in order to face them, extensibility becomes a further requirement for an IDS. The system should envisage the capability of easily adding new detection mechanisms or updating the existing ones, without compromising the correct operation of the system. Similarly, the IDS should be able to monitor different network segments, thus allowing for the seamless introduction of new observation points (i.e. new network probes). From the operational standpoint, an essential property of an IDS is also its capability to operate a real-time detection, in order to identify and react to intrusion attempts in a timely fashion. Finally, since the IDS is itself a component of the network, it might be victim of an attack. For this reason solutions aimed at increasing the intrinsic security of the system should be provided.

In this paper we present a distributed architecture which has been conceived starting from the above mentioned requirements. Such an architecture is comprised of a number of entities, each in charge of a specific task, and whose combined operation contributes to the effective identification of anomalous behavioral patterns within the analyzed network traffic.

This system has been developed as a component of a more complex framework designed within INTERSECTION ${ }^{1}$, a European research project on network security, which involves both academic and industrial partners. The main goal of INTERSECTION is to design an integrated security framework made of different subsystems and components, implementing diversity in terms of both analyzed data sources and employed analysis algorithms, capable of ensuring protection of heterogenous networks and infrastructures. The architecture described in this paper is currently used as one of the main components of a closed-loop network control system based on the concepts of detection, reaction and remediation. We will also present results and outcomes of the proposed solution's usage in the framework of the INTERSECTION network, spread across several academic and industrial project partners' network infrastructures.

The rest of the paper is organized as follows. In Section II we introduce the state of the art on intrusion detection systems. We present the proposed solution's architecture in Section III. In Section IV we present a formal analysis of the system's

\footnotetext{
${ }^{1}$ INTERSECTION Project - http://www.intersection-project.eu
} 
throughput and scalability. In Section $\mathrm{V}$ we present a use case for the proposed architecture in the framework of the INTERSECTION project. Detection performance evaluation is described in Section VI, and some conclusions are provided in Section VII.

\section{RELATED WORK}

Intrusion Detection Systems can be grouped into two main categories, depending on the monitored information, namely Network-based IDS (N-IDS) [3], and Host-based IDS (HIDS) [4]. The former analyze packets captured directly from the network, while the latter focus on a single host's activity, resulting from application logs and user based application usage. IDS can also be classified, based on the detection technique employed, in anomaly detection systems [5], which classify as anomalous all the events mismatching a welldefined "normal" model, and misuse detection systems [6], which classify as attacks all the events conforming to a well defined, and relatively static, model of anomalous behavior.

Recently, several solutions have been proposed to increase the effectiveness and the scalability of the detection process; in particular, distributed approaches have been investigated. In [7] the authors propose a scalable solution for high-performance intrusion detection by means of a cluster-based architecture. A scalable and flexible architecture for IDS is also presented in [8]. The authors design an innovative state management and migration framework that allows multiple network-IDS to exchange internal state information with the goal of providing each sensor with all the information that is necessary to perform a stateful traffic analysis. In [9] the author proposes a highly-configurable intrusion detection framework consisting of several autonomous agents which locally monitor network traffic and transfer collected intrusion detection information to a centralized data fusion server. The agents are extremely flexible since they can dynamically download and install appropriate modules, signatures and policy files from a central server. Another solution for intrusion detection based on agents is proposed in [10]. The proposed framework provides five different types of agents, each with a specific task, in order to analyze events from different sites, and to detect cases of attacks using an anomaly-based approach. An interesting solution is proposed in [11]. The authors present a parallel IDS: in spite of a canonical distributed IDS, such IDS is capable of monitoring traffic on a single ingress/egress point by performing discrete tasks, as data analysis, in a parallel fashion.

Our solution meets the main requirements stated above for an IDS. In spite of previous solutions the proposed architecture conjugates the main peculiarities of both distributed and parallel IDS. In particular, our framework provides a flexible solution allowing multi-point traffic data acquisition and analysis. Several analysis approaches, for example anomaly or misuse detection, can be exploited in parallel on the same traffic data set, thus improving the reliability of the overall detection process.

\section{A Distributed Architecture For Network INTRUSION DETECTION}

Detecting intrusions is a hard task in any networked environment, since a network naturally lends itself to a distributed exploitation of its resources. In such a scenario, the identification of a potential attack requires that information is gathered from many different sources and in many different places. Furthermore, current solutions lack a fundamental feature: dynamicity. A dynamic deployment of system resources and functionality should actually be required in order to ensure both flexibility of the architecture and robustness in the face of changes in network and traffic conditions.

For these reasons, we have chosen to design our architecture for intrusion detection as a distributed security system comprising a number of components, whose operation is orchestrated to ensure that the network is effectively protected. A distributed system, indeed, allows the separation of concerns among a well-defined set of entities, each conceived to deal with a particular aspect of the problem. This property on one hand simplifies the task of each involved entity, whereas on the other hand allows a deeper specialization of each module (which can thus be modified without necessarily affecting the performance of the overall system). In this section we will provide a high level description of both the system components and the communication protocol. Due to space limitations, the discussion will be focused on design choices rather than on detailed information about the implementation.

\section{A. Architecture Components}

The overall architecture of the IDS is depicted in Fig. 1. It consists of different modules specialized in specific tasks: (i) distributed IDS-Probes, which are in charge of monitoring traffic data in several points of the network, and extracting metrics from them; (ii) a Data Broker, which is in charge of gathering information from the monitoring system and distributing the collected information to one or more detection engines; (iii) distributed Detection Engines, which receive data from the broker and decide whether or not such information represents a potential attack pattern, based on a specific detection technique; (iv) a Decision Maker, which is in charge of combining information coming from multiple detection engines and analyzing diverse subsets of the source data in order to effectively detect attacks. The IDS analyzes traffic metrics provided by the probes for identifying attacks evidences. Traffic metrics of interest need to be selected according to the traffic properties which are deemed to be able of exposing the symptoms of addressed attack types, thus making attack-related traffic flows easily separable from normal traffic. Whenever a suspicious activity is detected, the system generates an alert event, which is meant to be sent to other INTERSECTION framework components.

Detection effectiveness depends on the technique employed for classification, as well as on both the traffic data analyzed and the metrics chosen to represent such data for the purpose of attack detection. Furthermore, the more suitable the metrics 


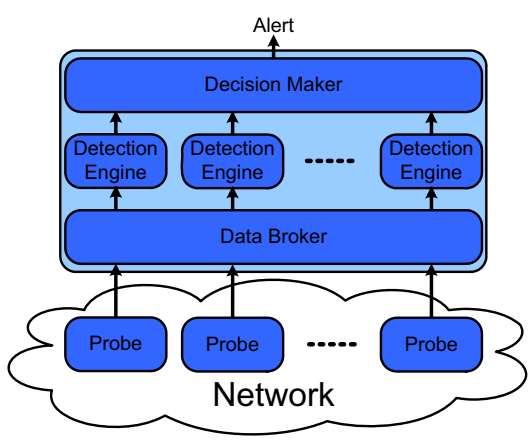

Fig. 1. The IDS Architecture

are, the more effective the separation between normal and anomalous traffic is.

A possible deployment of the IDS is depicted in Fig. 2. As proposed in the INTERSECTION project, the IDS should be capable of operating in heterogeneous networked infrastructures: for example in Fig. 2 several probes are spread across different infrastructures, including wireless sensor networks and satellite communication networks. Metrics computed from sniffed network traffic are collected by the data broker, which represents a front-end for several detection engines, deployed within a logically separate and dedicated local network, which receive information to analyze. A decision maker finally combines all the results coming from detection engines. The IDSProbe has been designed to support the extraction of per-flow metrics from network traffic. In order to increase the capability of the system to discriminate between normal and anomalous traffic, the component provides solutions for implementing both "fine-grained" and "coarse-grained" metrics [12]. Finegrained metrics summarize information about the activities of a single flow, whereas coarse-grained metrics provide information about the correlation among several flows sharing common properties. The metrics computed are then exported periodically by using the IPFIX protocol [13].

Another degree of freedom in the system, which could dramatically affect its performance, is the choice of the detection techniques to employ. So far, we discussed the issue of classification accuracy. Indeed, such a choice can affect the system's reliability as well. Not to disregard this issue, a diversitybased approach to the selection of detection techniques might

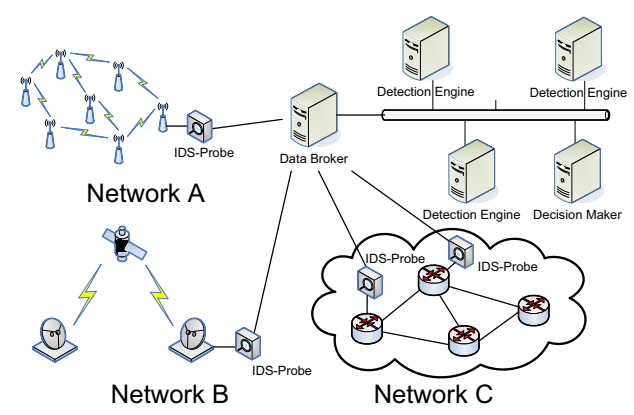

Fig. 2. An IDS Deployment be successfully exploited. In fact, by integrating different mechanisms for attack classification, the system may increase its detection capabilities, since more accurate analysis can be performed in order to detect specific attacks. A support for reliability evaluation mechanisms can be added when multiple detection techniques are used, thus allowing to estimate the accuracy of each issued decision. The proposed architecture easily allows to extend and integrate different mechanisms for attack classification at both the detection engine and decision maker sides. Any algorithm for data analysis can be implemented by a specific detection engine. In this way a new classification approach can be integrated in the system by implementing the proper detection engine. A specific protocol (see Subsection III-B) provides mechanisms for integration of any new module in the system. In some cases such an approach, exploiting several techniques, might require each technique to analyze different sets of monitored traffic metrics. This is because a detection process for a specific attack does not necessarily need to analyze all the network parameters provided by monitoring, but exclusively the parameters which are of interest to the specific anomaly or behavior it has been trained to detect. According with this observation the data broker is in charge of properly distributing the incoming traffic metrics to different detection engines.

In order to exploit the diversity of detection techniques we necessarily need also to define an effective approach for orchestrating and combining information coming from the multiple sources foreseen. In fact, a multiple classifier approach bases its performance improvement on the ability to effectively and securely detect attacks by combining information coming from diverse sources, each potentially using different detection techniques, and potentially analyzing distinct subsets of the source data. This task is performed by the decision maker by exploiting several techniques, ranging from very simple majority voting to complex information fusion. The decision maker response time is heavily influenced by the information expiration timeout, since the greater the timeout, the more information will be available for correlation, but the slower will be the detection. Such timeout is necessary in order to prevent the decision maker from waiting indefinitely for late information sent by the detection engines. This drawback, introduced by the usage of an unreliable protocol such as UDP, can be overcome by setting a limit to the time a decision maker is required to wait for missing information about observed events before taking an incomplete, yet timely decision.

\section{B. Communication Protocol}

The flexibility and the extendability of our architecture are largely due to the distribution of all the required tasks among several components spread within the network, and to a protocol which allows the entities to cooperate. More precisely, both a data protocol and a control protocol are needed. The data protocol must be characterized by a very limited overhead, both due to the nature of the problem, and to the place at which data to be analyzed are exchanged; the loss of data messages, in fact, is less harmful than the 
huge overhead which would be introduced by retransmissions and reassembly or reconstruction of corrupted and unordered packets. On the other hand, the control protocol is typically used for exchanging few and predictable messages, therefore requiring the support of a reliable transport protocol. For the mentioned reasons, we chose to use UDP for data exchange, and TCP for control messages exchange, in order to have unreliable yet lightweight communications in the former case, and reliable communications in the latter. Data exchange protocol messages are characterized by a field identifying the type and the identity of the component which originated the message, the type of conveyed message, as well as time-based information allowing to perform correlation and aggregation during further message processing stages.

In the following, the communication protocol adopted by our framework will be referred to as Control Protocol, and its description will be focused on its management aspects. The main control element in the architecture is the data broker; in addition to forwarding data flowing from the data feeds (e.g. the probes) to all active detection engines, it is responsible for orchestrating the synchronized behavior of the overall system. Supposing we have a working data broker with the management function active on it, we can regard it as a server ready to accept incoming subscription/disconnection requests. The whole architecture has to be thought of as a box where all the inputs contribute to the computation of the system's output. For this reason no detection engine can exist before a decision maker is subscribed to the aforementioned data broker. A DM_CONNECT subscription message sent by the decision maker will be confirmed with a CONNECTED message. The detection engine registration, by means of a DE_CONNECT message, allows the detection engine to specify which kind of data (such as the feature subset type) it will analyze, plus its connection preferences. This subscription request is followed by a synchronization message ( $\mathrm{SYNCH}$ ) sent by the broker to the decision maker, containing information about all the subscribed detection engines. The detection engine subscription is completed when it receives an acknowledgment (e.g. CONNECTED) message by the decision maker; such acknowledgement should contain decision maker connection preferences to receive alert messages from the detection engines. The IDS-Probes can subscribe by sending a SET_COMPONENTS request to the data broker, specifying the data set they will provide to the system; the acknowledgment will be realized by means of a TRANSMIT message, containing information for negotiating the type of backward data feed. Moreover, the data broker could dynamically modify the data flow's properties. The system allows detection engines to dynamically connect or disconnect from the architecture: to realize the disconnection, a detection engine is supposed to send an explicit request DE_DISCONNECT to the data broker; a DISCONNECT message will confirm the disconnection and a new SYNCH will be transmitted to the decision maker. Since the decision maker component is fundamental for the architecture's existence, its disconnection, by means of a DM_DISCONNECT message and confirmed by a DISCONNECT, causes the implicit dis- connection of all detection engines subscribed to the system. The IDS-Probe disconnection implies just a notification to the broker and its acknowledgment, without any synchronization messages. Figure 3 shows subscription message exchanges for all the components. In order to secure communication

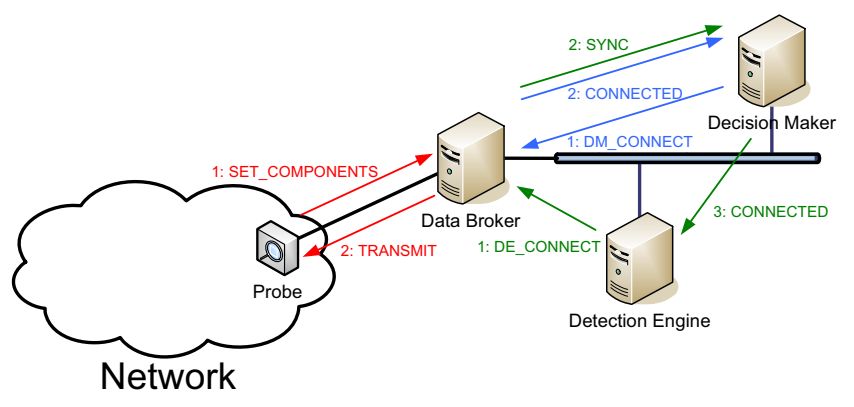

Fig. 3. IDS Control Protocol

between system components, the proposed architecture relies on authentication and secure encrypted communication channels implemented by using Secure Sockets Layer (SSL) and Datagram Transport Layer Security (DTLS). Certificates and shared secrets are typically exchanged before subscription. In a typical deployment configuration, it is affordable to encrypt and authenticate information flowing in control channels, whereas data channel encryption becomes soon unbearable when the traffic rate increases, therefore making the choice of an encrypted data exchange unfeasible.

\section{SYSTEM ANALYSIS}

In this section we provide a qualitative analysis of detection reliability, as well as scalability and extendability of our proposed solution. In Section VI we will then evaluate the system performance on a real networking scenario. Usually, a multiple classifier system is at least as accurate as the best of its base classifiers [1]. In this paper, which mainly addresses architectural issues, we only implement a majority voting algorithm, aimed at demonstrating the correctness of the approach from a functional point of view; better suited and more accurate combination approaches will be proposed in future work. Given a set of classifiers whose individual decisions are combined by using a simple majority voting mechanism, a necessary and sufficient condition for increasing the reliability of a multiple classifier system, with respect to a single classifier, is that the individual classifiers are accurate and diverse enough [1]. The classifiers are accurate and diverse if they have, respectively, an error rate lower than 0.5 , and their errors are independent. In such hypotheses, assuming that the $M$ base classifiers all exhibit the same error probability $p$, the error probability for a multiple classifier system based on the majority voting combination mechanism can be represented in terms of a binomial distribution; such a multiple classifier system is wrong if more than $M / 2$ base classifiers are wrong:

$$
\sum_{k>M / 2}^{M}\left(\begin{array}{c}
M \\
k
\end{array}\right) p^{k}(1-p)^{M-k}
$$


For example, for 5 classifiers, each characterized by error probability 0.2 , the overall error probability is 0.05792 . The error probability function described by equation (1) monotonically decreases with $M$, which assures that by increasing the number of the base classifiers, the overall classification accuracy increases.

In order to evaluate the scalability of the architecture we propose a qualitative analysis based on the properties of queueing networks theory. We consider the scalability of the framework as its capability to operate efficiently, even as the overall system load increases, by suitably increasing the number of probes and detection engines. In Fig. 4 we

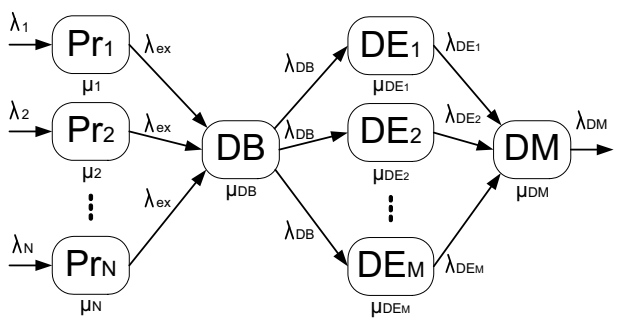

Fig. 4. IDS Scalability Analysis

represent the IDS architecture by means of a network of queues, where each component is characterized by the message arrival rate and the service rate. We assume that the system is composed of $N$ probes, deployed within the network, and $M$ detection engines for data analysis. In order to simplify our formulation, we introduce some restrictive hypotheses. First, we assume a fixed service rate $\mu_{D E}$ for all the detection engines. Such an assumption does not considerably influence our analysis. Furthermore we disregard the probes in our model, since they are characterized by a constant exporting time $T_{e x}=1 / \lambda_{e x}$, independent of the arrival rate, which is influenced by traffic rate, and service rate, depending on the selected metric computation algorithms. From queueing theory, any component works correctly, and its queue's size doesn't diverge, if it satisfies the following constraint:

$$
\rho=\frac{\lambda}{\mu}<1
$$

where $\rho$ is the utilization factor. The efficiency of the data broker, of the detection engines and the decision maker is assured if, in turns:

$$
\frac{\lambda_{P r_{t o t}}}{\mu_{D B}}=\frac{N \cdot \lambda_{e x}}{\mu_{D B}}<1 ; \frac{\lambda_{D B}}{\mu_{D E}}<1 ; \frac{\lambda_{D E_{t o t}}}{\mu_{D M}}=\frac{M \cdot \lambda_{D E}}{\mu_{D M}}<1
$$

or, equivalently, if

$$
\lambda_{e x}<\frac{\mu_{D B}}{N} ; \lambda_{D B}<\mu_{D E} ; \lambda_{D E}<\frac{\mu_{D M}}{M}
$$

The arrival rate at every component, if the utilization factor satisfies the stability constraint, is related to the arrival rate of components in the previous stage, i.e. $\mu_{D B}=\lambda_{D B}$ and $\mu_{D E}=\lambda_{D E}$. By exploiting equation (4) we can determine the bottleneck of our architecture through the following relation:

$$
\mu_{D B}>N \cdot \lambda_{e x} ; \mu_{D E}>N \cdot \lambda_{e x} ; \mu_{D M}>N \cdot M \cdot \lambda_{e x}
$$

As shown by equation (5), the decision maker's computation capability represents the architecture's bottleneck. The service rate DM imposes a constraint on the number of probes and detection engines that the framework can support. Such a limit can be overcome by increasing the number of data brokers and decision makers in the architecture. Without violating the consistency of either the data or the analysis process, we can consider, for example, two data brokers and two decision makers. Each data broker manages a set of probes, and, by exploiting all the detection engines, the classification results are collected by a well known decision maker. This is possible since each detection engine can be subscribed to different data brokers and decision makers. Let us consider $K$ data brokers collecting data from a set of $N=K$ probes, and $K$ corresponding decision makers. The constraints in equation (3) become:

$$
\frac{\frac{N}{K} \cdot \lambda_{e x}}{\mu_{D B}}<1, \frac{\lambda_{D B}}{\mu_{D E}}<1, \frac{M \cdot \lambda_{D E}}{\mu_{D M}}<1
$$

By exploiting the same formulation, in particular

$$
\mu_{D M}>\frac{M \cdot N \cdot \lambda_{e x}}{K}
$$

In this way, by increasing the number of data brokers and decision makers we can reduce the service rate $\mu_{D M}$ needed to support $N$ probes and $M$ detection engines. Equation (7) assures that, if we increase either the number $N$ of probes or the number $M$ of detection engines we can still have an efficient system by adding new data brokers and decision makers, thus satisfying the scalability requirement for our architecture. Given the exporting rate $\lambda_{e x}$, the elaboration time of each components $1 / \mu$, the number of probes $N$ and detection engines $M$, it is also possible to determine the number $K$ of data brokers and decision makers needed by using equation (7).

\section{A Demo Case: DDos Attack Detection}

As mentioned above, our IDS solution has been developed and deployed in the context of the INTERSECTION project as a component of a more complex framework designed for the detection of various attacks in heterogeneous network infrastructures. In order to demonstrate the effectiveness of the approach several demonstration cases have been designed, which involve a prototype of the overall INTERSECTION framework for the detection and remediation of attacks. In this section we focus on the demo scenario aimed at proving Distributed Denial of Service (DDoS) attack detection. Such demo scenario, which was one of the main outcomes of the project, directly involved the network IDS as a fundamental component.

This demo case required the IDS deployment across a distributed testbed made of several project partners' interconnected laboratories. GRE tunnels have been established among geographically distributed sites in order to create an extended virtual private network. The realized testbed is shown in Fig. 5, where also a list of involved project partners is given. A typical DDoS attack scenario involves several components, 


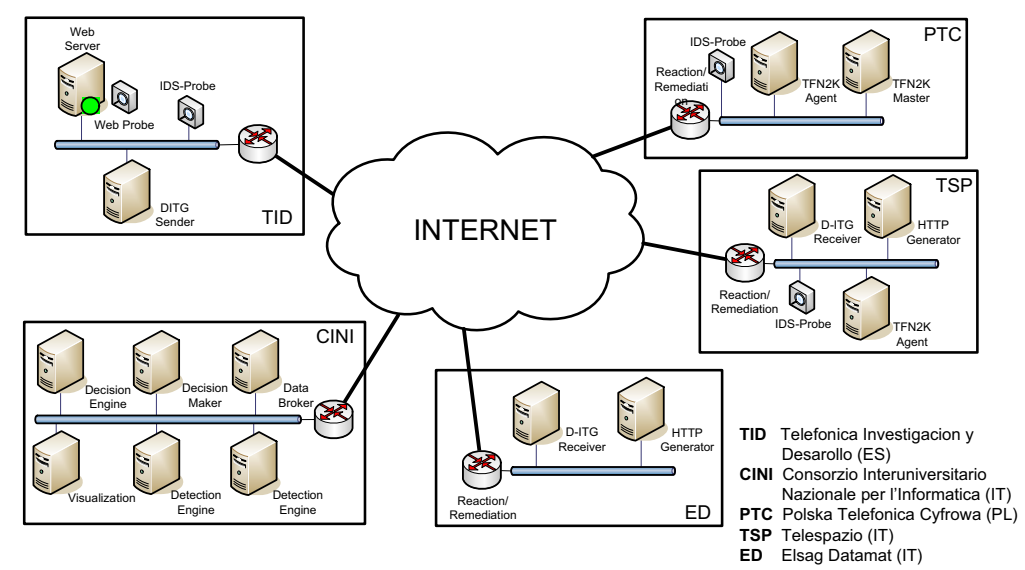

Fig. 5. DDoS Demo Scenario

potentially distributed throughout large portions of the network, comprising: a "master", who initiates and orchestrates the distributed attack, several "agents", or "zombies", which receive commands from the master and perform the actual attack activities, and a target node, which represents the victim of the attack. Once the victim has been identified, the attacker launches the attack by waking up the master host. The master communicates with the agents in order to instruct them about the attack to perform against the target by means, for example, of common packet flooding procedures. In this demo scenario we assume that each agent participates in SYN-Flooding attack targeting the victim host.

The overall detection process is performed by monitoring the system at two different levels: (i) network based monitoring, performed by our architecture which analyzes traffic activities, and alerts the Decision Engine if anomalies are detected, and (ii) host-level probes which monitor the target machine by controlling operating system parameters and identify anomalous values of such parameters, which may be symptoms of the ongoing attack. The Decision Engine, included in the INTERSECTION architecture (Fig. 5), correlates these two sources of alerts and detects the SYN-Flooding attack. Then, it sends an alarm to two other components of the INTERSECTION prototype, namely the Reaction and the Visualization modules (Fig. 5).

In order to implement this demo scenario we used the TFN2K ${ }^{2}$ tool for the DDoS attack. TFN2K consists of two modules: a command driven client to be installed on the master host and a daemon program for the agent host. TFN2K exploits four different flooding mechanisms for attacking the target, including SYN-Flooding attack. As shown in Fig. 5 the DDoS master node is hosted at the PTC lab, together with an agent node, while another agent is hosted at the TSP site. Actually, each machine hosting an agent can run several instances of the daemon, in order to increase the amount of traffic generated for the attack. The target, instead, just consists of a host running an APACHE HTTP server ${ }^{3}$ at the TID lab. In order to realize a

\footnotetext{
${ }^{2}$ http://packetstormsecurity.org/distributed/TFN\$2\$K.tgz

${ }^{3}$ http://httpd.apache.org/
}

more realistic scenario we considered the generation of some background traffic. We adopted HAMMERHEAD ${ }^{4}$ to generate HTTP traffic targeting the web-server. In a real scenario we expect HTTP traffic to flow from multiple IP domains towards a single destination. For this reason we emulated multiple sources by installing two generators at both the TSP and ED sites, and by exploiting the IP aliasing mechanism at each machine in order to have a larger number of network addresses. We also generated different types of background network traffic from the local network where the web-server is installed to the public network (as an example of traffic from a campus network to the Internet). We used the D-ITG traffic generator ${ }^{5}$, which allows to generate several traffic profiles, including VoIP traffic. D-ITG requires a sender module, which we installed at the TID lab and at least a receiver module; we installed two D-ITG receivers at TSP and ED, respectively. In order to approximate a realistic scenario we again used multiple source addresses and multiple destination addresses by exploiting the IP aliasing mechanism on each machine.

The demo scenario requires several IDS-Probes disseminated throughout the network in order to effectively observe the evidences of a distributed attack, whereas all the other IDS components are hosted at CINI lab (Fig. 5). As mentioned above in this section, the demo scenario also included other components of the INTERSECTION prototype: the Decision Engine, the Visualization module at the CINI lab, a Web probe installed onboard the HTTP server, and several Reaction/Remediation modules at the ingress nodes of each laboratory. The IDS-Probes calculate traffic metrics similar to the metrics defined in [14]. In particular, each single probe provides, for each analyzed packet: (i) the duration of the connection which the packet belongs to (for UDP packets such duration is conventionally equal to zero) (duration), (ii) the number of connections during the last 2 seconds having the same destination host as the current packet (count), and (iii) the percentage of connections to the same destination host with the same source port as the current packet

\footnotetext{
${ }^{4} \mathrm{http}: / /$ sourceforge.net/projects/hammerhead/

5 toolhttp://www.grid.unina.it/software/ITG/
} 
(dst_host_same_src_port_rate). These three metrics succeed in detecting a DDoS attack in our specific scenario, since they show high discrimination capability between normal and attack traffic. The selection of these features among the available feature set has been accurately performed by means of a feature selection process based on sequential forward selection.

During the demo we first generated background traffic by establishing data flows between traffic generation modules at the TSP, ED and TID networks. Then the attack was started at the PTC site, where the master commanded the local agent and the agent at the TSP lab to flood the victim hosted at the TID lab. IDS-Probes have proven able to register these flooding activities, and send metrics to the Data Broker at the CINI lab for the analysis. The monitoring data are forwarded to several Detection Engines, whose analysis results are collected by the Decision Maker. The final verdict is sent by the Decision Maker to the Decision Engine for a high level alert correlation. At the final project demo, the whole prototype components' functionality has been tested, and the IDS components, together with the other modules, have been proven capable of detecting, and reacting to the performed DDoS attack.

\section{PERformance Evaluation}

In section $\mathrm{V}$ we have described the deployment of the proposed IDS in a real network scenario. In that scenario, all the IDS components were able to interact correctly; the whole architecture has proven capable of successfully detecting $\mathrm{DDoS}$ attack instances, thus demonstrating the correct implementation of the desired functionality, the compliance with the requirements, and the fulfillment of the project's claims. In this section, indeed, we want to study detection performance in further detail, introducing several diverse detection engines, and evaluating the detection performance on a per-packet basis, in order to obtain an indication of how precise and how effective the system can really be.

In order to define the overall performance of the proposed IDS, each component has been tested both in terms of packet loss ratio, with respect to an increasing traffic rate, and traversal time, aimed at measuring how much time a data unit spends inside the IDS. Furthermore, the capability of the system to properly identify attacks has been evaluated. More in details, packet loss ratio is evaluated on a per-component basis. For the Data Broker, we evaluate the ratio between metrics packets sent to each subscribed Detection Engine and metrics packets sent by the Probes; for the Detection Engines, we evaluate the ratio between the number of detection results computed with respect to the number of metrics packets sent by the Data Broker; for the Decision Maker, the ratio between the number of analyzed alerts and the number of alerts sent by the Detection Engines is computed. Results about the Data Broker performance evaluation are depicted in Fig. 6, and show that the component drops less than $5 \%$ of the packets for an incoming traffic rate greater than $30 \mathrm{Mb} / \mathrm{s}$. In the same conditions, Fig. 7 shows that the Detection Engines

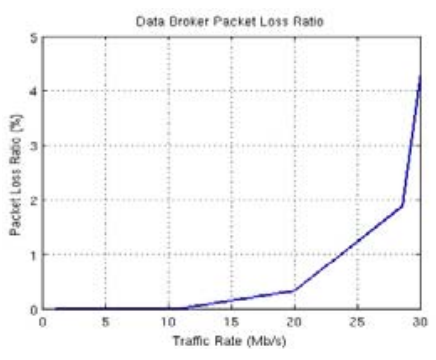

Fig. 6. Data Broker Loss Ratio

drop less than $1.4 \%$ of incoming traffic. The Decision Maker's performance figure is omitted, since it doesn't show any packet dropping for an input rate as high as $30 \mathrm{Mb} / \mathrm{s}$. It is worth pointing out that the overall performance is evaluated as a worst case boundary, since for the described measurements we use the whole set of available metrics. In a realistic scenario, the most suitable metrics can be chosen after a reasonable training period, therefore further reducing the network and computational load resulting from IDS operations. As to the traversal time, it has been evaluated as the time taken by the system to compute a final decision, at the Decision Maker side, about a metrics packet received, instants before, at the Broker side. In particular, it has been measured that each packet takes about 2 milliseconds from the time it enters the Data Broker buffer until it is sent to all subscribed Detection Engines. The output produced by the Data Broker feeds the Detection Engines, where a less significant delay is experienced: it takes less than 2 milliseconds between queueing at the Detection Engine input buffer and completing the detection procedure required by the analysis algorithms. Finally, the decision maker service time evaluation is meaningfully affected by the configured timeout for final decision: if the Decision Maker doesn't receive detection information from all the subscribed Detection Engines, it waits until such timeout expires before making its own decision. Hence, the overall system performance has been strongly influenced by the probe's capabilities and the decision maker's timer, which are operational choices strictly dependent on the configuration parameters related to specific operational environment's characteristics.

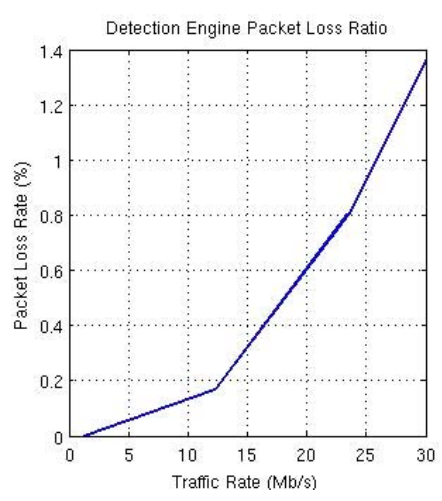

Fig. 7. Detection Engine Loss Ratio 
We also evaluated the performance of our solution in terms of detection accuracy. The main objective of this investigation has been the evaluation of the multiple classifier approach adopted for system design in terms of robustness to detection errors. In the following, we will refer to metrics evaluating the false positive, false negative, true positive and true negative rates. During these tests, we evaluated detection accuracy on a per packet basis, whereas during the demo presented in section $\mathrm{V}$ we evaluated the ability to detect an attack instance, the ability of the components to perform functionally correct interactions, and the capability of the overall prototype, including the network IDS as a macro component, to appropriately react to a DDoS attack in an heterogeneous network scenario. We setup a controlled testbed to generate traffic traces used for extracting traffic models through computational intelligence algorithms, which can be later exploited by the detection engines for on-line traffic evaluation. Arrays of metrics elaborated off-line have been analyzed by several supervised machine learning algorithms for extracting a traffic model. Seven different algorithms of different nature (TABLE I) have been used for implementing base classifiers. Each model is implemented in a detection engine through a set of rules. Detection engines' results feed the decision maker, which combines them by means of a majority voting mechanism; detection results are reported in the last row of the table. As to the results shown in TABLE I, the overall system's detection performance stays as good as the best of the base classifiers. Indeed, the multiple classifier system implemented by our IDS is able to compensate occasional very poor detection rates, such as the ones shown by the SVM-based classifier on the analyzed dataset of this example. In general, poor detection capability may depend on the ever-changing nature of network traffic, on the selected features and on how suitable the implemented classification technique is. A multiple classifier system can help compensate all these undesirable, yet very likely to occur shortcomings. Furthermore, due to increasing traffic rate, and to the inherent increase in traffic monitoring complexity, scalability properties shown in previous section make a distributed system a good design choice, given an acceptable trade-off with overall detection performance.

TABLE I

IDS PERFORMANCE

\begin{tabular}{|c|c|c|c|c|}
\hline & f.p. & f.n. & t.p. & t.n. \\
\hline Support Vector Machine & 0.002 & 1.000 & 0.000 & 0.999 \\
\hline Multi Layer Perceptron & 0.000 & 0.086 & 0.952 & 1.000 \\
\hline J48 Decision Tree & 0.004 & 0.020 & 0.980 & 0.997 \\
\hline Conjunctive Rule & 0.000 & 0.020 & 0.980 & 1.000 \\
\hline Bayesian Network & 0.000 & 0.323 & 0.677 & 1.000 \\
\hline Bagging & 0.001 & 0.020 & 0.980 & 0.999 \\
\hline AdaBoost & 0.003 & 0.020 & 0.980 & 0.997 \\
\hline Multiple Classifier IDS & 0.000 & 0.020 & 0.980 & 1.000 \\
\hline
\end{tabular}

\section{CONCLUSIONS}

In this paper we have presented a distributed architecture for flexible and reliable real-time intrusion detection. The rationale behind our proposal resides in the observation that a parallel, multi-classification approach can in many cases improve the overall detection capabilities of an intrusion detection system. We have carried out an analysis of the performance of the architecture in terms of scalability and extensibility, as well as capability to operate in real-time, and detection accuracy. Solutions for the authentication of the components during the subscription phase have been investigated and implemented, together with optionally configurable secure communication channels encrypting the whole control and detection messages exchange.

\section{ACKNOWLEDGMENTS}

The research leading to these results has received funding from the European Community's Seventh Framework Programme (FP7/2007-2013) under Grant Agreement No. 216585 (INTERSECTION Project) and Grant Agreement No. 225553 (INSPIRE Project).

\section{REFERENCES}

[1] L. K. Hansen and P. Salamon, "Neural network ensembles," IEEE Transactions on Pattern Analysis and Machine Intelligence, vol. 12, no. 10, pp. 993-1001, 1990.

[2] T. G. Dietterich, "Ensemble methods in machine learning," Lecture Notes in Computer Science, vol. 1857, pp. 1-15, 2000.

[3] M. Roesch, "Snort - lightweight intrusion detection for networks," in 13th USENIX conference on System administration, 1999, pp. 229-238.

[4] R. Moskovitch, S. Pluderman, I. Gus, D. Stopel, C. Feher, Y. Parmet, Y. Shahar, and Y. Elovici, "Host based intrusion detection using machine learning," in IEEE Intelligence and Security Informatics, May 2007, pp. $107-114$.

[5] D. Mutz, F. Valeur, G. Vigna, and C. Kruegel, "Anomalous system call detection," ACM Transactions on Information and System Security, vol. 9, no. 1, pp. 61-93, 2006.

[6] W. Tylman, "Misuse-based intrusion detection using bayesian networks," in Third International Conference on Dependability of Computer Systems, 2008, pp. 203-210.

[7] M. Vallentin, R. Sommer, J. Lee, C. Leres, V. Paxson, and B. Tierney, "The nids cluster: Scalable, stateful network intrusion detection on commodity hardware," in International Symposium on Recent Advances in Intrusion Detection (RAID), September 2007, pp. 107-126.

[8] M. Colajanni, D. Gozzi, and M. Marchetti, "Enhancing interoperability and stateful analysis of cooperative network intrusion detection systems," in ACM/IEEE Symposium on Architecture for Networking and Communications Systems, December 2007, pp. 165-174.

[9] Y. Cai, "A distributed autonomous intrusion detection framework," in IEEE Globecom Workshop, November 2007, pp. 1-5.

[10] D. Boughaci, H. Drias, A. Bendib, Y. Bouznit, and B. Benhamou, "Distributed intrusion detection framework based on autonomous and mobile agents," in IEEE International Conference on Dependability of Computer Systems, May 2006, pp. 248-255.

[11] P. Wheeler and E. Fulp, "A taxonomy of parallel techniques for intrusion detection," in ACM Southeast Regional Conference, March 2007, pp. 278-282.

[12] S. D'Antonio, M. Esposito, F. Oliviero, S. P. Romano, and D. Salvi, "Behavioral network engineering: making intrusion detection become autonomic," Annals of Telecommunications, vol. 61, no. 9-10, pp. 11391151, 2006.

[13] B. Claise, "Rfc 5101 - specification of the ip flow information export (ipfix) protocol for the exchange of ip traffic flow information," IETF," Internet Draft, January 2008.

[14] W. Lee and S. J. Stolfo, "A framework for constructing features and models for intrusion detection systems," ACM Transactions on Information and System Security, vol. 3, no. 4, pp. 227-261, November 2000. 